\title{
Rastreamento de sinais e sintomas de transtornos do espectro do autismo em irmãos
}

\author{
Screening for signs and symptoms of autism spectrum disorders in siblings \\ Tatiana Pontrelli Mecca ${ }^{1}$, Riviane Borghesi Bravo², Renata de Lima Velloso ${ }^{3}$, José Salomão Schwartzman
Decio Brunoni ${ }^{4}$, Maria Cristina Triguero Veloz Teixeira ${ }^{6}$
}

${ }^{1}$ Psicóloga. Doutoranda, Programa de Pós-Graduação em Distúrbios do Desenvolvimento, Centro de Ciências Biológicas e da Saúde, Universidade Presbiteriana Mackenzie, São Paulo, SP. ${ }^{2}$ Psicóloga. Mestre, Programa de Pós-Graduação em Ciências da Saúde: Saúde da Criança e do Adolescente, Faculdade de Medicina, Universidade Federal de Minas Gerais (UFMG), Belo Horizonte, MG. ${ }^{3}$ Doutoranda, Programa de Pós-Graduação em Distúrbios do Desenvolvimento, Centro de Ciências Biológicas e da Saúde, Universidade Presbiteriana Mackenzie. ${ }^{4}$ Doutor. Professor titular, Programa de Pós-Graduação em Distúrbios do Desenvolvimento, Centro de Ciências Biológicas e da Saúde, Universidade Presbiteriana Mackenzie. ${ }^{5}$ Doutor. Professor titular, Programa de Pós-Graduação em Distúrbios do Desenvolvimento, Centro de Ciências Biológicas e da Saúde, Universidade Presbiteriana Mackenzie. ${ }^{6}$ Doutora. Professora adjunta I, Programa de Pós-Graduação em Distúrbios do Desenvolvimento, Centro de Ciências Biológicas e da Saúde, Universidade Presbiteriana Mackenzie.

\section{Resumo}

Os transtornos globais do desenvolvimento (TGD) são caracterizados por anormalidades qualitativas e abrangentes em três domínios do desenvolvimento: interação social recíproca, comunicação e presença de um repertório comportamental de interesses restritos, repetitivo e estereotipado. Estudos genéticos têm identificado a recorrência de TGD numa mesma família. O presente estudo teve por objetivo rastrear a ocorrência de sinais e sintomas de TGD em irmãos de indivíduos com esse diagnóstico. Participaram do estudo 25 sujeitos provenientes de 19 famílias. A coleta de dados foi realizada mediante a utilização da versão brasileira do Autism Screening Questionnaire (ASQ), ou Questionário de Comportamento e Comunicação Social. Foram confirmados dois casos de irmãos com TGD (10,52\% dos casos), sendo um irmão gêmeo monozigótico e um irmão de um probando com diagnóstico de síndrome de Asperger. Os dados apontam para taxas mais elevadas do que aquelas descritas na literatura (2-6\%) e se aproximam dos achados que relatam $10 \%$ de recorrência familiar em gêmeos dizigóticos. Esse resultado fornece evidências de possíveis fatores neurogenéticos para explicar a ocorrência de TGD nos familiares dos probandos estudados e salienta a necessidade de efetuar o rastreamento desse transtorno não só na criança avaliada, mas também em seus irmãos.

Descritores: Irmãos, genética, transtornos do espectro do autismo.

\begin{abstract}
Pervasive developmental disorders (PDD) are characterized by comprehensive and qualitative abnormalities affecting three areas of development: reciprocal social interaction, communication, and a repetitive, stereotyped behavioral repertoire, of limited interests. Genetic studies have identified the recurrence of PDD in the same family. The present study aimed to trace the occurrence of signs and symptoms of PDD in the siblings of patients with this diagnosis. The study included 25 subjects from 19 families. Data collection was performed using the Brazilian version of the Autism Screening Questionnaire (ASQ). Two cases of PDD in siblings were confirmed (10.52\% of cases): a monozygotic twin brother and the brother of a proband with a diagnosis of Asperger syndrome. Our data indicate higher rates of PDD in siblings than described in the literature (2-6\%), close to the findings that suggest a $10 \%$ rate of familial recurrence in dizygotic twins. This result provides evidence of possible neurogenetic factors to explain the occurrence of PDD in relatives of the probands assessed and underscores the need to screen not only the child under evaluation, but also their siblings.
\end{abstract}

Keywords: Siblings, genetics, autism spectrum disorders.

\section{Correspondência:}

Maria Cristina Triguero Veloz Teixeira, Programa de Pós-Graduação em Distúrbios do Desenvolvimento, Centro de Ciências Biológicas e da Saúde, Universidade Presbiteriana Mackenzie, Rua da Consolação, 896, prédio 38, térreo, Consolação, CEP 01302-907, São Paulo, SP. E-mail: cris@teixeira.org

Não foram declarados conflitos de interesse associados à publicação deste artigo.

Copyright (C) Revista de Psiquiatria do Rio Grande do Sul - APRS

Recebido em 10/02/2010. Aceito em 23/09/2010. 


\section{Introdução}

Atualmente, o autismo está classificado na categoria dos transtornos globais do desenvolvimento (TGD) ou transtornos invasivos do desenvolvimento em manuais de classificação, tais como o Diagnostic and Statistical Manual of Mental Disorders, 4th edition, Text Revision (DSM-IV-TR) e a Classificação Estatística de Doenças e Problemas Relacionados à Saúde - $10^{\mathrm{a}}$ Revisão (CID-10), respectivamente ${ }^{1,2}$. O diagnóstico se estabelece a partir desses critérios clínicos, que abrangem a identificação de anormalidades em três domínios do desenvolvimento: interação social recíproca, comunicação e presença de um repertório comportamental de interesses restritos, repetitivo e estereotipado.

De acordo com o DSM-IV-TR ${ }^{2}$, os TGD se classificam em transtorno autista, transtorno de Rett, transtorno desintegrativo da infância, transtorno de Asperger e transtorno global do desenvolvimento sem outra especificação (TGD-SOE). Entretanto, perante a complexidade da condição, nem sempre os casos avaliados preenchem todos os critérios diagnósticos para o transtorno autista. Por esse motivo, atualmente, o grupo de trabalho da Associação Americana de Psiquiatria do DSM-V discute critérios clínicos diferentes e a criação de uma nova categoria diagnóstica para incluir o autismo. O grupo propõe excluir da condição de TGD o transtorno desintegrativo da infância e a síndrome de Rett, e incluir os três transtornos restantes na condição transtornos do espectro do autismo (TEA). Esta última se subdividiria em duas subcategorias: TEA tipo I, para casos típicos do espectro autista; e TEA tipo II, para casos atípi$\cos ^{3}$.

O fenótipo dos TEA é heterogêneo e, embora apresente uma etiologia multifatorial, é o único transtorno neuropsiquiátrico no qual se tem mostrado considerável influência da herdabilidade ${ }^{4-12}$. Essa influência hereditária tem conduzido à realização de diversos estudos para identificar alterações neurocomportamentais em irmãos de crianças diagnosticadas com TEA ${ }^{13-17}$.

Os primeiros estudos, que apresentaram taxas de recorrência entre 90 e $96 \%$ para gêmeos monozigóticos e de 0 a 24\% em dizigóticos, datam dos anos 1980 e $1990^{18-20}$. Estudos familiares com gêmeos que abrangem a identificação de anormalidades cromossômicas fornecem evidências de que vários genes em interação podem contribuir para esse transtorno ${ }^{21}$. Pesquisas que identificaram risco de recorrência de autismo entre gêmeos monozigóticos mostraram uma taxa superior a $90 \%$ quando se considera o amplo espectro de manifestações ${ }^{18-20,22,23}$. Um estudo de revisão recente $^{12}$ mostrou pesquisas que apontam taxas de concordância em gêmeos monozigóticos variando de 73 a $95 \%$ e um risco de 5 a $6 \%$ de recorrência em irmãos. Em relação à recorrência familiar, um estudo realizado por Sumi et al. ${ }^{24}$ mostrou taxa de $10 \%$ para recorrência familiar em irmãos de indivíduos diagnosticados com TEA.
Para identificar a ocorrência familiar dos TEA em irmãos, são utilizados preferencialmente escalas e inventários de rastreamento ${ }^{24-26}$. Esses instrumentos têm algumas vantagens, entre elas o custo relativamente baixo associado a seu uso e sua elevada sensibilidade para identificar alterações clínicas do espectro do autismo.

Na última década, aumentou no Brasil o número de pesquisas destinadas à tradução e adaptação de instrumentos para rastreamento e auxílio no diagnóstico dos TEA ${ }^{27-}$ 32. Por outro lado, estudos sobre a ocorrência familiar do transtorno são praticamente inexistentes no país, assim como são escassas as pesquisas de cunho epidemiológico. Dessas últimas, apenas dois estudos epidemiológicos foram divulgados até o momento ${ }^{33,34}$. Já os trabalhos que focam outras áreas, como diagnóstico, marcadores neurogenéticos, aspectos psicossociais, entre outros, são mais frequentes ${ }^{28,35-44}$. Assim, avaliações diagnósticas desse tipo, realizadas pelas equipes de saúde, poderiam ser ampliadas para familiares de primeiro grau. Sugere-se, para isso, o uso de instrumentos adaptados culturalmente no país ${ }^{28-32}$.

Desta forma, o objetivo deste estudo foi identificar sinais e sintomas de TEA em irmãos de indivíduos previamente diagnosticados com o transtorno.

\section{Método}

O estudo foi composto por uma série de casos de 25 irmãos de 19 crianças e adolescentes com diagnóstico de TGD. Entre as 19 pessoas afetadas, oito tinham diagnóstico de transtorno autista, sete apresentavam transtorno de Asperger e quatro tinham TGD-SOE. Todos os diagnósticos foram estabelecidos por equipe multidisciplinar composta por especialistas em neuropediatria, genética, psicologia, fonoaudiologia e pedagogia, mediante uso dos critérios estabelecidos no DSM-IV-TR ${ }^{2}$.

Os 25 irmãos foram identificados em 19 famílias, cuja triagem foi feita através dos registros existentes nos protocolos dos pacientes da Clínica de Distúrbios do Desenvolvimento, Programa de Pós-Graduação em Distúrbios do Desenvolvimento, Universidade Presbiteriana Mackenzie, em São Paulo (SP). Foram selecionadas famílias nas quais os probandos possuíam um ou mais irmãos. As famílias foram contatadas e convidadas a participar da pesquisa. Todos os irmãos dos probandos participaram do estudo. Entre os pacientes estudados, foram descartados os que apresentavam quadros sindrômicos de causa genética ou ambiental.

A coleta de dados foi realizada mediante a aplicação da versão brasileira do Autism Screening Questionnaire (ASQ), ou Questionário de Comportamento e Comunicação $S_{0}$ cial ${ }^{32}$. O instrumento é composto por 40 questões que abrangem três domínios, a saber, interação social, linguagem e comportamentos, tendo por objetivo rastrear sinais e sintomas dos TEA. O ASQ foi aplicado às mães dos 
pacientes com TGD. Os procedimentos de análise de dados foram estabelecidos de acordo com as normas para pontuação, sendo previstas três classificações: 0 a 14 pontos (normal); 15 a 21 pontos (TGD); e > 21 (autismo).

O estudo teve aprovação do Comitê de Ética em Pesquisa da universidade (protocolo $n^{\circ}$ 081/05/07 e CAAE0031.0.272.000-07).

\section{Resultados}

Dos 25 irmãos, 23 obtiveram pontuações abaixo da linha de corte $(89,48 \%)$, um irmão $(5,26 \%)$ teve pontuação compatível com TGD e um irmão $(5,26)$ teve pontuação compatível com o diagnóstico de autismo. O caso que obteve pontuação para TGD no ASQ era irmão gêmeo monozigótico de 8 anos de idade do probando. $\mathrm{O}$ segundo caso classificado como autismo era irmão de uma criança diagnosticada com síndrome de Asperger. Esses resultados apontam para um índice de $10,52 \%$ de ocorrência de sinais e sintomas de TGD na amostra estudada.

A aplicação do ASQ permitiu observar diferentes graus de prejuízo nas três áreas investigadas. A Tabela 1 apresenta as pontuações obtidas nas áreas de comportamentos restritos, repetitivos e estereotipados, comunicação/linguagem e interação social.

De acordo com a pontuação obtida no ASQ para as três áreas e também a pontuação total, observa-se que os prejuízos na interação social obtiveram pontuações elevadas nos dois casos identificados com transtorno autista e TGD (sujeitos 9 e 22).

Tabela 1 - Pontuações obtidas nas áreas de comportamento, comunicação/linguagem e interação social do ASQ

\begin{tabular}{|c|c|c|c|c|c|c|}
\hline Sujeito & Família & Condição & ASQ & Comportamento & $\begin{array}{c}\text { Comunicação/ } \\
\text { linguagem }\end{array}$ & $\begin{array}{c}\text { Interação } \\
\text { social }\end{array}$ \\
\hline 1 & 1 & Irmão DZ & 2 & 1 & 1 & 0 \\
\hline 2 & 2 & Irmã & 7 & 3 & 3 & 1 \\
\hline 3 & 3 & Irmão & 1 & 1 & 0 & 0 \\
\hline 4 & 3 & Irmão & 5 & 1 & 1 & 3 \\
\hline 5 & 4 & Irmã & 1 & 0 & 0 & 1 \\
\hline 6 & 5 & Irmã & 1 & 0 & 0 & 1 \\
\hline 7 & 6 & Irmão & 8 & 4 & 2 & 2 \\
\hline 8 & 6 & Irmã & 1 & 0 & 1 & 0 \\
\hline 9 & 7 & Irmão MZ & $19 *$ & 7 & 5 & 7 \\
\hline 10 & 8 & Irmã & 4 & 0 & 4 & 0 \\
\hline 11 & 9 & Irmã & 6 & 0 & 6 & 0 \\
\hline 12 & 10 & Irmã & 0 & 0 & 0 & 0 \\
\hline 13 & 10 & Irmão & 0 & 0 & 0 & 0 \\
\hline 14 & 11 & Irmã & 4 & 1 & 2 & 1 \\
\hline 15 & 12 & Irmã & 0 & 0 & 0 & 0 \\
\hline 16 & 13 & Irmã & 2 & 0 & 2 & 0 \\
\hline 17 & 13 & Irmão & 3 & 0 & 2 & 1 \\
\hline 18 & 14 & Irmão & 9 & 1 & 6 & 2 \\
\hline 19 & 14 & Irmã & 13 & 1 & 7 & 5 \\
\hline 20 & 15 & Irmão & 0 & 0 & 0 & 0 \\
\hline 21 & 15 & Irmã & 2 & 0 & 2 & 0 \\
\hline 22 & 16 & Irmão & $32 *$ & 9 & 10 & 13 \\
\hline 23 & 17 & Irmão & 10 & 1 & 6 & 3 \\
\hline 24 & 18 & Irmão & 5 & 0 & 4 & 1 \\
\hline 25 & 19 & Irmão & 7 & 0 & 3 & 4 \\
\hline
\end{tabular}

ASQ = Autism Screening Questionnaire; $\mathrm{DZ}=$ dizigótico; $\mathrm{MZ}=$ monozigótico.

* Sujeitos que pontuaram para TGD e autismo no ASQ. 


\section{Discussão}

Apesar da amostra restrita de 25 irmãos, os achados apontaram sinais e sintomas para TEA em dois casos da série. Esse resultado coincidiu, em termos percentuais, com os achados de Ritvo et al. ${ }^{45}$, que, ao estudar 207 famílias, encontraram uma taxa de $9,6 \%$ de recorrência em irmãos.

Se comparada com estudos recentes que apontam índices de recorrência familiar de TEA entre 2 e $6 \%$, é provável que a taxa de ocorrência familiar encontrada em nossa amostra tenha sido mais elevada devido a uma limitação própria do trabalho, a saber, a de ter analisado uma amostra composta apenas por irmãos de uma série de pacientes cuja seleção não seguiu critérios probabilísticos ${ }^{46-48}$. Por exemplo, Lintas \& Persico, em estudo de revisão sistemática, apontaram como risco de recorrência em irmãos uma taxa de $5-6 \%{ }^{12}$. Uma taxa mais próxima aos nossos achados foi encontrada por Sumi et al., com $10 \%$ de recorrência em irmãos ${ }^{24}$

Vale ressaltar que os achados do presente trabalho devem ser considerados com cautela ao serem generalizados à população. O estudo pretende apenas alertar, preferencialmente psiquiatras, neurologistas e pediatras, sobre a necessidade de avaliar familiares, especialmente irmãos, quando diagnosticarem algum caso afetado por TEA.

No Brasil, é de extrema necessidade o desenvolvimento de programas direcionados para a detecção precoce de TEA. O trabalho agrega informações que poderão servir de alerta às equipes de saúde, principalmente pediatras. São eles os profissionais que identificam, em crianças, indicadores de diversos tipos de alterações comportamentais, entre as quais estão os TEA. Com uma atuação clínica focada em familiares e probandos, será possível implementar estratégias mais amplas de manejo e orientação familiar quando necessário.

\section{Conclusões}

Os presentes dados permitem recomendar às equipes de saúde que avaliam crianças com suspeita de TEA que também realizem, sempre que possível, o rastreamento do transtorno em irmãos dos probandos. Os achados focaram sinais clínicos do distúrbio; entretanto, recomenda-se ampliar e diversificar, em diversas amostras brasileiras, a existência de manifestações do espectro do autismo em irmãos de afetados. Nos casos positivos, intensa investigação genética deve ser iniciada com o intuito de afastar casos sindrômicos do distúrbio.

A aplicação de protocolos amplos de maneira sistemática no estudo de casos de indivíduos com TEA deve prever a investigação genealógica e a aplicação de questionários de rastreamento de sinais e sintomas em consanguíneos dos probandos, principalmente irmãos. Tal estratégia permitirá conhecimento detalhado das manifestações desse transtorno nas famílias, propiciando melhor assistência às mesmas.

\section{Referências}

1. Organização Mundial da Saúde. Transtornos mentais e comportamentais. In: Classificação Estatística de Doenças e Problemas Relacionados à Saúde - $10^{\mathrm{a}}$ Revisão. $8^{\mathrm{a}}$ ed. São Paulo: Edusp; 2000.

2. American Psychiatric Association. Diagnostic and Statistical Manual of Mental Disorders (DSM-IV-TR). Washington: APA; 2003.

3. First MB. Autism and Other Pervasive Developmental Disorders Conference (February 3-5, 2008). Arlington: American Psychiatric Association; 2008. http://www.psych.org/MainMenu/Research/DSMIV/DSMV/DSMRevisionActivities/ConferenceSummaries/AutismConference.aspx. Acessado jan 2010.

4. Carvalheira G, Vergani N, Brunoni D. Genética do autismo. Rev Bras Psiquiatr. 2004;26(4):270-2

5. Santangelo SL, Tsatsanis K. What is known about autism: genes, brain, and behavior. Am J Pharmacogenomics. 2005;5(2):71-92.

6. Kelemenova S, Ostatnikova D. Neuroendocrine pathways altered in autism. Special role of reelin. Neuro Endocrinol Lett. 2009;30(4):429-36.

7. Levy SE, Mandell DS, Schultz RT. Autism. Lancet. 2009;374(9701):1627-38.

8. Pardo-Govea T, Solís-Añez E. Immunogenetic aspects of autism. Invest Clin. 2009;50(3):393-406.

9. Autism and Developmental Disabilities Monitoring Network Surveillance Year 2006 Principal Investigators; Centers for Disease Control and Prevention (CDC). Prevalence of autism spectrum disorders - Autism and Developmental Disabilities Monitoring Network, United States, 2006. MMWR Surveill Summ. 2009;58(10):1-20.

10. Weiss LA, Arking DE. A genome-wide linkage and association scan reveals novel loci for autism. Gene Discovery Project of Johns Hopkins \& the Autism Consortium. Nature. 2009;461(7265):802-8.

11. Matuszek G, Talebizadeh Z. Autism Genetic Database (AGD): a comprehensive database including autism susceptibility gene-CNVs integrated with known noncoding RNAs and fragile sites. BMC Med Genet. 2009;10:102.

12. Lintas C, Persico AM. Autistic phenotypes and genetic testing: state-of-the-art for the clinical geneticist. J Med Genet. 2009;46:1-8.

13. Garon N, Bryson SE, Zwaigenbaum L, Smith IM, Brian J, Roberts W, et al Temperament and its relationship to autistic symptoms in a high-risk infant sib cohort. J Abnorm Child Psychol. 2009;37(1):59-78.

14. Ibanez LV, Messinger DS, Newell L, Lambert B, Sheskin M. Visual disengagement in the infant siblings of children with an autism spectrum disorder (ASD). Autism. 2008;12(5):473-85.

15. Yirmiya N, Gamliel I, Pilowsky T, Feldman R, Baron-Cohen S, Sigman M The development of siblings of children with autism at 4 and 14 months: social engagement, communication, and cognition. J Child Psychol Psychiatry. 2006; 47(5):511-23.

16. Muhle R, Trentacoste SV, Rapin I. The genetics of autism. Pediatrics. 2004;113(5):472-86

17. Zhao X, Leotta A, Kustanovich V, Lajonchere C, Geschwind DH, Law K, et al A unified genetic theory for sporadic and inherited autism. Proc Natl Acad Sci U S A. 2007;104(31):12831-6.

18. Ritvo E, Freeman BJ, Manson-Brothers AMA, Ritvo AM. Concordance for the syndrome of autism in 40 pairs of afflicted twins. Am J Psychiatry. 1985;142:74-7.

19. Steffenburg S, Gillberg C, Hellgren L, Andersson L, Gillberg IC, Jakobsson G, et al. A twin study of autism in Denmark, Finland, Iceland, Norway and Sweden. J Child Psychol Psychiatry. 1989;30:405-16.

20. Bailey A, Le Counteur A, Gottesman I, Bolton P, Simonoff E, Yuzda E, et al Autism as a strongly genetic disorder: evidence from a British twin study. Psychol Med. 1995;25:63-77.

21. Acosta MT, Pearl PL. Aspectos genéticos do autismo. In: Tuchman R, Rapin I Autismo: abordagem neurobiológica. Porto Alegre: Artmed; 2009.

22. Jamain $S$, Betancur $C$, Giros $B$, Leboyer $M$, Bougeron $T$. La génétique de l'autisme. Med Sci (Paris). 2003;19(11):1081-90.

23. Gupta AR, State MW. Autismo: genética. Rev Bras Psiquiatr. 2006;28(suppl 1):29-38.

24. Sumi S, Taniai H, Miyachi T, Tanemura M. Sibling risk of pervasive developmental disorder estimated by means of an epidemiologic survey in Nagoya, Japan. J Hum Genet. 2006;51:518-22.

25. Constantino JN, Lajonchere C, Lutz M, Gray T, Abbacchi A, McKenna K, et al Autistic social impairment in the siblings of children with pervasive developmental disorders. Am J Psychiatry. 2006;163(2):294-6.

26. Taniai H, Nishiyama T, Miyachi T, Imaeda M, Sumi S. Genetic influences on the broad spectrum of autism: study of proband-ascertained twins. Am J Med Genet B Neuropsychiatr Genet. 2008;147B(6):844-9.

27. Williams JG, Higgins JP, Brayne CE. Systematic review of prevalence studies of autism spectrum disorders. Arch Dis Child. 2006;91(1):2-5. 
28. Marteleto MR, Pedromônico MR. Validity of Autism Behavior Checklist (ABC): preliminary study. Rev Bras Psiquiatr. 2005;27(4):295-301.

29. Aguiar CLC. A tradução da ADI-R, Entrevista Diagnóstica de Autismo - Revisada. São Paulo: Universidade Presbiteriana Mackenzie; 2005.

30. Lopásio MF, Pondé MP. Tradução para o português da escala M-CHAT para rastreamento precoce de autismo. Rev Psiquiatr Rio Gd Sul. 2008;30(3):221-9.

31. Pereira A, Riesgo RS, Wagner MB. Childhood autism: translation and validation of the Childhood Autism Rating Scale for use in Brazil. J Pediatr (Rio J). 2008;84(6):487-94.

32. Sato FP, Paula CS, Lowenthal R, Nakano E, Brunoni D, Schwartzman JS, et al. Instrument to screening cases of pervasive developmental disorder: a preliminary indication of validity. Rev Bras Psiquiatr. 2009;31(1):30-3.

33. Duarte CS, Bordin IA, Yazigi L, Mooney J. Factors associated with stress in mothers of children with autism. Autism. 2005;9(4):416-27.

34. Lowenthal R, Paula CS, Schwartzman JS, Brunoni D, Mercadante MT. Prevalence of pervasive developmental disorder in Down's syndrome. J Autism Dev Disord. 2007;37(7):1394-5.

35. Steiner CE, Guerreiro MM, Marques-de-Faria AP. Genetic and neurological evaluation in a sample of individuals with pervasive developmental disorders. Arq Neuropsiquiatr. 2003;61(2A):176-80.

36. Lampreia C. Avaliações quantitativa e qualitativa de um menino autista: uma análise crítica. Psicol Estud. 2003;8(1):57-65.

37. Araújo CAS. A perspectiva winnicottiana sobre o autismo no caso de Vitor. Psyche (Sao Paulo). 2004;8(13):43-60

38. Fontenelle LF, Mendlowicz MV, Bezerra de Menezes G, dos Santos Martins RR, Versiani M. Asperger syndrome, obsessive-compulsive disorder, and major depression in a patient with $45, \mathrm{X} / 46, \mathrm{XY}$ mosaicism. Psychopathology. 2004;37(3):105-9.
39. Oliveira AB, Giunco CT, Carvalho AB, Salles F, Conte ACF. Investigação molecular por PCR da síndrome do X-frágil em homens com transtorno invasivo do desenvolvimento. Arq Cienc Saude. 2004;11(1):25-8.

40. De Leon C. Psychological Profile Revised (PEP-R): the Brazilian version elaboration. Autism. 2005;9(4):450-2.

41. Steiner CE, Guerreiro MM, Marques-de-Faria AP, Lopes-Cendes I. Laboratorial diagnosis of fragile- $\mathrm{X}$ syndrome: experience in a sample of individuals with pervasive developmental disorders. Arq Neuropsiquiatr. 2005;63(3A):564-70.

42. Mercadante MT, Macedo EC, Baptista PM, Paula CS, Schwartzman JS. Saccadic movements using eye-tracking technology in individuals with autism spectrum disorders: pilot study. Arq Neuropsiquiatr. 2006;64(3A):559-62.

43. Mugno D, Ruta L, D'Arrigo VG, Mazzone L. Impairment of quality of life in parents of children and adolescents with pervasive developmental disorder. Health Qual Life Outcomes. 2007;5:22.

44. Stachon A, Assumpção Júnior FB, Raskin S. Rett syndrome: clinical and molecular characterization of two Brazilian patients. Arq Neuropsiquiatr. 2007;65(1):36-40.

45. Ritvo ER, Jorde LB, Mason-Brothers A, Freeman BJ, Pingree C, Jones MB, et al. The UCLA-University of Utah Epidemiologic Survey of Autism: recurrence risk estimates and genetic counseling. Am J Psychiatry. 1989;156(8):1032-6.

46. Chakrabarti S, Fombonne E. Pervasive developmental disorders in preschool children. JAMA. 2001;27:3093-9.

47. Icasiano F, Hewson P, Machet P, Cooper C, Marchall A. Childhood autism spectrum disorder in Barwon region: a community based study. J Paediatr Child Health. 2004;40:696-701

48. Newschaffer CJ, Croen LA, Daniels J, Giarelli E, Grether JK, Levy S, et al. The epidemiology of autism spectrum disorders. Annu Rev Public Health. 2007;28:235-58 